\title{
Does Patent Foramen Ovale Presence Procure Favourable Outcomes In Patients With Severe Rheumatic Mitral Stenosis?
}

\section{Gamze Guler ( $\sim$ gamzebabur@hotmail.com )}

Mehmet Akif Ersoy Thoracic and Cardiovascular Research and Education Hospital: Mehmet Akif Ersoy Gogus Kalp Damar Cerrahisi Egitim Arastirma Hastanesi

\section{Arda Can Dogan}

Mehmet Akif Ersoy Thoracic and Cardiovascular Research and Education Hospital: Mehmet Akif Ersoy Gogus Kalp Damar Cerrahisi Egitim Arastirma Hastanesi

\section{Ali Kemal Kalkan}

Mehmet Akif Ersoy Thoracic and Cardiovascular Research and Education Hospital: Mehmet Akif Ersoy Gogus Kalp Damar Cerrahisi Egitim Arastirma Hastanesi

\section{Ali Riza Demir}

Mehmet Akif Ersoy Thoracic and Cardiovascular Research and Education Hospital: Mehmet Akif Ersoy Gogus Kalp Damar Cerrahisi Egitim Arastirma Hastanesi

\section{Begum Uygur}

Mehmet Akif Ersoy Thoracic and Cardiovascular Research and Education Hospital: Mehmet Akif Ersoy Gogus Kalp Damar Cerrahisi Egitim Arastirma Hastanesi

\section{Ali Birant}

Mehmet Akif Ersoy Thoracic and Cardiovascular Research and Education Hospital: Mehmet Akif Ersoy Gogus Kalp Damar Cerrahisi Egitim Arastirma Hastanesi

\section{Hicaz Zencirkiran Agus}

Mehmet Akif Ersoy Thoracic and Cardiovascular Research and Education Hospital: Mehmet Akif Ersoy Gogus Kalp Damar Cerrahisi Egitim Arastirma Hastanesi

\section{Maria Concetta Pastore}

University of Siena: Universita degli Studi di Siena

\section{Kadriye Memic}

Mehmet Akif Ersoy Thoracic and Cardiovascular Research and Education Hospital: Mehmet Akif Ersoy Gogus Kalp Damar Cerrahisi Egitim Arastirma Hastanesi

\section{Fatih Uzun}

Mehmet Akif Ersoy Thoracic and Cardiovascular Research and Education Hospital: Mehmet Akif Ersoy Gogus Kalp Damar Cerrahisi Egitim Arastirma Hastanesi

\section{Matteo Cameli}

Università degli Studi di Siena: Universita degli Studi di Siena 
Mehmet Erturk

Mehmet Akif Ersoy Thoracic and Cardiovascular Research and Education Hospital: Mehmet Akif Ersoy Gogus Kalp Damar Cerrahisi Egitim Arastirma Hastanesi

\section{Research Article}

Keywords: Mitral stenosis, patent foramen ovale, left atrial pressure

Posted Date: March 17th, 2021

DOl: https://doi.org/10.21203/rs.3.rs-304813/v1

License: (c) (1) This work is licensed under a Creative Commons Attribution 4.0 International License. Read Full License 


\section{Abstract}

Purpose:Mitral stenosis (MS) is torelated for an extended period in patients with atrial septal defect (ASD) known as Lutembacher syndrome due to depressurizing effect. In a similar way, patients with patent foramen ovale (PFO) may have clinical benefits in severe MS. We aim to evaluate the clinical effects of PFO in rheumatic MS.

Methods and Results:Transthoracic and transesophageal echocardiography records of the patients with symptomatic severe MS were screened for the period between 2008-2019 in a single center. Threehundred-twenty symptomatic patients with severe MS were included and presence of PFO recorded. Left atrial appendix (LAA) thrombotic status was defined as clear, spontaneous echo contrast, and thrombus. Two different statistical models were used to determine the predictors of either smallest (mitral valve area) MVA at symptomatic presentation or more thrombogenic LAA. Thirty-four patients had PFO. Multivariable ordinary least square model demonstrated that increase in systolic pulmonary arterial pressure, ejection fraction and presence of PFO were associated with smaller MVA on presentation. Multivariable proportional odds logistic regression model demonstrated that advanced age, increased left atrial diameter, absence of PFO were associated with more thrombotic status whereas larger MVA was associated with decreased thrombotic status in LAA.

Conclusion:Presence of PFO in severe MS results in two clinical benefits as i) being asymptomatic with smaller MVA and ii) having less LAA thrombosis probably caused by depressurizing effect on the left atrial pressure. Our study, could serve as an example for patient groups with expected symptomatic benefits from left atrium pressure offloading interventions.

\section{Introduction:}

Rheumatic mitral valve stenosis (MS) pertains its place as acquired form valvular pathology and still results in morbidity and mortality. Increased left atrial pressure results in Delay in diastolic emptying, decrease in cardiac output ${ }^{1}$,increase in left atrial pressure, pulmonary venous hypertension and superimposed arterial hypertension ${ }^{2}$ play a role in pathophysiological events which cause patients to be symptomatic. Lutembacher described the Lutembacher syndrome in 1916 referred to his own name, which consists of MS accompanied by an atrial septal defect (ASD) $)^{3}$. In general, it is known that the MS is more benign and patients tolerate MS better for a longer time periods when ASD is also present ${ }^{3}$.

Reduced left atrial appendage (LAA) functions in rheumatic MS were documented both in spectral and tissue Doppler echocardiography studies ${ }^{4,5}$. Decreased contraction of LAA causes spontaneous echo contrast (SEC) and thrombus formation even in MS patients with sinus rhythm ${ }^{5-7}$.

Patent foramen ovale (PFO) is an interatrial septum abnormality which is detected in $27.3 \%$ of the normal population in autopsy series ${ }^{8}$. In a transesophageal echocardiography (TEE) study, PFO with left to right 
shunt was detected in $27 \%$ in left-sided cardiac lesions and it was speculated that this served to offload left atrial pressure referring to Lutembacher syndrome like physiology ${ }^{9}$.

Based on these pathophysiological phenomenon, practice of percutaneous implantation of shunt devices to the interatrial septum have been added introduced as a nonpharmacological treatment alternative for heart failure. It was reported that these devices reduce left atrium (LA) pressure, particularly during exercise, and increase patient's exercise capacity ${ }^{10}$.

Based on our clinical observations we hypothesized that 1-Patients had smaller valve areas at symptomatic presentation,2-In the presence of PFO, thrombus and SEC in the LAA were observed fewer. Our primary focus was to investigate whether patients with PFO had a smaller mitral valve area (MVA) when admitted with symptoms. Secondary aim was to assess the relationship between thrombus formation frequency in the LAA and presence of a PFO. This retrospective study was designed to investigate whether concomitant presence of PFO makes a positive contribution to clinical presentation in two different pathways in severe MS patients by screening the TEE images in our hospital imaging database.

\section{Methods}

\section{Study population}

It is a single-center, retrospectively designed study. Medical records of the patients who underwent TEE due to symptomatic severe MS between 2008-2019, were screened. The patients with MVA $<1.5 \mathrm{~cm}^{2}$ and at most mild to moderate mitral regurgitation (MR) or aortic regurgitation (AR) were included. The patients who had MVA $>1.5 \mathrm{~cm}^{2}$, moderate or severe MR or AR, tricuspid stenosis, severe pulmonary stenosis and regurgitation, left ventricle ejection fraction $(\mathrm{LVEF})<50 \%$, previous history of percutaneous mitral balloon valvuloplasty (PMBV), ASD, partial and total pulmonary venous return anomalies, moderate or severe aortic stenosis were excluded.

After evaluation of these criteria, 320 patients with severe MS who underwent transthoracic echocardiography (TTE) and TEE to evaluate suitability for PMBV were included in the study. Transesophageal and transthoracic echocardiography reports and TEE images archived to Picture Archiving and Communication Systems (PACS) system were reevaluated.

The study was approved by the local ethics committee.

The study protocol is in line with the Declaration of Helsinki ${ }^{11}$.

\section{Electrocardiography}

Electrocardiographies (ECG) found in the medical records were revisisted. Atrial fibrillation was defined by the absence of a P wave and irregular QRS complexes on 12-lead surface ECG.

Echocardiographic measurements 
Echocardiography reports were screened and PACS TEE images of patients included in the study were reassessed. A standardized TEE protocol (19) was used to define valvular disease and atrial septum morphology.

Rheumatic MS evaluation was performed according to the European Association of Echocardiography and American Society of Echocardiography recommendations for echocardiographic assessment of valve stenosis ${ }^{12}$.

Anatomical evaluation of the valve includes presence of commissural fusion (assessed with 2D and 3D echocardiography), leaflet thickness and motion, chordal shortening and thickening, presence of fibrotic nodules and calcification and extension (Fig. 1A). Continuous Doppler wave was used in apical views for mitral flow. Through this measurement, mean gradient was calculated by tracing diastolic mitral flow contour; pressure half time (PHT) was measured from E wave descending slop and MVA was calculated. With 3D echocardiography, the narrowest orifice measurement was made with the multiplanar reconstruction (MPR) method in which the valve was evaluated from three orthogonal planes (Fig. 1B). Planimetric (PLN) valve area measurement was made by determining the narrowest orifice by scanning from the apex to the base, from the $2 \mathrm{D}$ parasternal short axis at mid-diastole containing the commissures (Fig. 1C). Average values obtained from 3-5 beats were used for patients in atrial fibrillation. Systolic pulmonary arterial pressure (SPAP) was calculated using the maximum velocity of the tricuspid regurgitant jet and estimated right atrial pressure. Wilkins score was calculated by evaluating the valve mobility, thickness, calcification extent and thickness of the subvalvular apparatus.

Quantification of valvular regurgitations was performed according to recommendations for the echocardiographic assessment of native valvular regurgitation ${ }^{13}$. Mild MR was defined as vena contracta $(\mathrm{VC})<3 \mathrm{~mm}$, effective regurgitant orifice area $(E R O A)<20 \mathrm{~mm}^{2}$, regurgitant volume $(\mathrm{RV})<30 \mathrm{ml}$; mild to moderate MR was defined as VC intermediate ( $3 \mathrm{~mm}<\mathrm{VC}<7 \mathrm{~mm}$ ), EROA 20-29 mm², RV 30-44 ml. The VC was measured by optimizing gain/scale and from two orthogonal planes where the 3 components of the regugitant jet (VC, proximal isovelosity surface area (PISA), jet into LA) were identified. For EROA and $\mathrm{RV}$ calculation, PISA radius was measured at mid-systole using the first aliasing at the appropriate Nyquist limit. EROA and RV were calculated using peak MR velocity and tissue velocity imaging. Mild AR was defined as VC $<3 \mathrm{~mm}, \mathrm{EROA}<10 \mathrm{~mm}^{2}$, RV $<30 \mathrm{ml}$; mild to moderate AR was defined as VC intermediate ( $3 \mathrm{~mm}<\mathrm{VC}<6 \mathrm{~mm}$ ), EROA 10-19 $\mathrm{mm}^{2}, \mathrm{RV} 30-44 \mathrm{ml}$. Mild tricuspid regurgitation (TR) was defined as PISA radius $<5 \mathrm{~mm}$, small, central jet; moderate TR was defined as PISA radius 6-9mm; severe TR was defined as $\mathrm{VC}>7 \mathrm{~mm}$, PISA radius $>9 \mathrm{~mm}$, EROA $\geq 40 \mathrm{~mm}^{2}, \mathrm{RV} \geq 45 \mathrm{ml}$.

PFO (Fig. 2) was defined as a potential space or separation between the septum primum and secundum at the anterosuperior portion of the atrial septum, and a " stretched " PFO was present when atrial hemodynamics have enlarged the foramen and resulted in left to right or right to left shunting seen on Doppler imaging, depending on the differences in the right and LA pressure. ${ }^{14}$ 
The SEC was recorded when dynamic smoke like echoes with characteristic rotational movement, at an appropriate gain setting, were seen. LAA thrombus was defined as the presence of echogenic mass, which is distinctly different from pectinate muscles.

\section{Clinical data}

The patients' hypertension, diabetes mellitus, cerebrovascular event (CVA) records were obtained from the institutional electronic medical records system (HBYS: hospital information management system) and archive files. Neurology clinic attendance and history of transient ischemic attack or cerebrovascular accident were recorded. Acetyl salicylic acid and/or warfarin use was screened in medical records of patients.

\section{Statistical analyses}

All statistical analyzes were performed using "MASS", "rms", "ggplot2" and "ggridges" packages with Rsoftware v. 3.5.1 (R statistical software, Institute for statistics and mathematics, Vienna, Austria). The continuous variables were presented as mean $\pm \mathrm{SD}$ and median (interquartile range (IQR)) and as percentage (number of cases) for categorical variables.

The primary outcomes were; i) Continuous MVA and ii) LAA thrombotic ordinal status (0:clear, 1:SEC, 2:thrombus). Accordingly, we have built two multivariable regression models;

1. Multivariable ordinary least square model was built with continuous MVA as the outcome of interest. Covariates included in the model were: SPAP, LVEF, LA diameter, gender, MR grades and PFO. Effects of individual predictors on MVA were reported by using regression coefficient and $95 \%$ confidence interval (Cl).

2. Multivariable proportional odds logistic regression model was built with LAA thrombotic ordinal status (0: clear, 1: SEC, 2: thrombus) as the outcome of interest. Covariates included in the model were: Age, MVA, LA diameter, LVEF, gender, MR grades, baseline anti-thrombotic drugs and PFO. The Brant test was used to test the parallel regression assumption, and none of the covarites violated the assumption. Effects of individual predictors on LAA thrombus were reported by using common Odds ratio and $95 \% \mathrm{Cl}$.

To allow for nonlinear associations, continuous predictors were modeled by using restricted cubic spline transformations. Regression coefficient or Odds ratio were presented change from 25th to 75th percentile for continuous variables. The relative importance of each predictor in the models was estimated with partial $R^{2}$ / partial $X^{2}$ value for each predictor divided by the model's total partial $R^{2} / X^{2}$, which estimates the independent contribution of the predictor to the variance of the outcome (partial $R^{2}$ for least square and $\mathrm{X}^{2}$ for ordinal regression).

\section{Results}


We enrolled retrospectively 320 patients in accordance with the inclusion criteria. The mean age was 48 \pm 12 years and $75(23,4 \%)$ were male. Mean MVA (PHT) was $1,15 \pm 0,19 \mathrm{~cm}^{2}$, mean MVA (PLN) was 1,15 $\pm 0,19 \mathrm{~cm}^{2}$, and mean MVA 3D MPR was $1,09 \pm 0,23 \mathrm{~cm}^{2}$. Median mean gradient and maximum gradients for rheumatic MS were 13, (IQR:16-9) mmHg and 21, (IQR:26-17) mmHg, respectively. The calculated median Wilkins scores of patients evaluated before PMBV or mitral valve replacement was 8 , (IQR:9-7). Thirty-four (10,6\%) patients had PFO. While 121 patients were in atrial fibrillation, 190 had sinus rhythm. From patients in sinus rhythm 22 were under warfarin treatment compared to 66 in the atrial fibrillation group. A total of $88(27,5 \%)$ patients were on warfarin, and $81(25,3 \%)$ were on ASA treatment. LAA thrombotic status was classified into three groups classified as no thrombus or SEC (166 patients, $51.9 \%)$, SEC (81 patients, $25.3 \%$ ) and thrombus (72 patients, $22.5 \%)$. Thirty-one (10,5\%) cerebrovascular events with no significant difference between PFO and no PFO groups were observed. Clinical characteristics of patients included are shown in Table 1.

Multivariable ordinary least square model demonstrated that increase in SPAP, LVEF and presence of PFO were associated with decrease in MVA at presentation (Table 2). We showed the relative importance of each predictor in the regression model. SPAP, LVEF and PFO were the strongest predictors for MVA at symptomatic presentation and caused approximately $90 \%$ of explainable MVA variance.

Multivariable proportional odds logistic regression model demonstrated that increase in age and LA diameter, absence of PFO were associated with more favorable thrombotic status of LAA whereas large MVA resulted in less frequent thrombosis in LAA (Table 3). There was an acceptable discrimination with a c-index of 0.737 . Age, LA diameter, MVA and PFO were the strongest predictors for MVA at symptomatic presentation and were responsible for approximately $90 \%$ of explainable LAA thrombotic ordinal status variance.

\section{Discussion}

In this study, two different outcomes of PFO presence were assessed retrospectively in a patient cohort with severe rheumatic MS. Firstly, MS patients with PFO were found to have smaller MVAs at symptomatic presentation. In addition SPAP and LVEF were identified as the additional predictors of smaller MVA at presentation. When severe MS was accompanied by a PFO, LAA thrombus or SEC frequency was significantly lower. Other predictors of LAA thrombotic status were smaller MVA, advanced age and dilatation of LA. To the best of our knowledge, this is the first study reporting the hemodynamic contribution of PFO presence on the clinical variance in MS.

PFO is a congenital interatrial septum abnormality which is not uncommon to persist in adult life and usually has an asymptomatic course ${ }^{15}$. A prevalence study investigating PFO frequency in patients with left-sided cardiac lesions (majority of the patients had MS) emphasized that reaching the critical LA pressure caused a small shunt that would disappear spontaneously after the correction of index lesion ${ }^{9}$. PFO shunts can be seen in both directions and this is determined by pressure gradient between left and 
right atrium. PFO shunt usually enables the high pressure to be decompressed without increasing the ventricular workload ${ }^{9}$.

Congenital or iatrogenic defects of the interatrial septum in patients with increased LA and RA pressures provide insight for this phenomenon. Interatrial balloon septostomy ${ }^{16}$ or IAS fenestration ${ }^{17}$ have been incorporated into practice in severe pulmonary hypertension $(\mathrm{PH})$ as one of the non-pharmacological treatment options to offload RA pressure. The rationale of decompressing the LA was inspired by the more benign course of MS in Lutembacher syndrome, and outcomes are associated with the diameter of the ASD as well as the degree of pulmonary vascular resistance ${ }^{3,18}$. In a case series published by Steinbrunn et al, cases with atrial shunt (mostly ASD) accompanying MS were hemodynamically evaluated and isolated PFOs were suggested to be secondary to LA stretch. They also observed that, patients with ASD had no paroxysmal dyspnea or pulmonary edema attacks. When worsening of pulmonary congestion after ASD closure were investigated in two patients, they concluded that interatrial shunt decompressed LA and pulmonary venous system protectively ${ }^{18}$. Also, in iatrogenic ASDs occurring after PMBV; absence of newly developing or worsening pulmonary hypertension ${ }^{19}$ and follow-up Qp/Qs of $<1.5^{20}$ pioneered in determining the safety margin for this procedure.

Increasing LA pressure with exercise constitutes the cornerstone in both the symptomatology and the functional capacity in the heart failure with prevented ejection fraction (HFPEF) patient group 21,22 .In the REDUCE LAP-HF study, after implantation of intracardiac shunt device in HFPEF patients, the exercise capacity and quality of life increased and peak pulmonary capillary wedge pressure decreased ${ }^{23}$. Simulation studies reported that the $8 \mathrm{~mm}$ iatrogenic defect is sufficient to decrease LA pressure without significant right ventricular volume load by keeping the Qp/Qs value around 1.3-1.4 $4^{10}$. Additinally, this device was shown to achieve improvement in pulmonary functional tests, both at rest and exercise, without impairment of systemic perfusion in a patient group with $\mathrm{HFPEF}^{24}$.

As discussed previously, although there are many pathological factors determining the symptomatology in severe MS, LA pressure increase constitutes the cornerstone. In the interventional or surgical treatment algorithm, pulmonary arterial hypertension secondary to increased pulmonary venous pressure also has an important role. We excluded patients with moderate-severe MR from our study population to eliminate the confounding effect of concomitant LA volume overload that can be seen in this patients.

In this patient group PFO opens a gateway for LA volume emptying so the patient gains the area of PFO diameter in addition to the MVA to offload the left atrium. Therefore, we would also expect the LA diameter to be smaller patients with PFO, but there was no statistical difference in our patient group. Based on our findings and physiologically it seems reasonable for MS patients to remain asymptomatic for a longer periods and to present with smaller valve area. Pediatric cardiologists know that, in a newborn with congenital mitral stenosis when PFO coexists, the gradient will be relatively low underestimating the stenosis. With this study, we showed that described physiological phenomenon might be of clinical benefit for our adult patient group with PFO and severe MS. 
Interestingly, while assessing the predictors of smaller MVA at symptomatic presentation, LVEF has made a significant contribution even within normal limits. Although patients with LVEF $<50 \%$ were excluded, 'more' normal EF and increase in EF from 60 to 65 have resulted in a statistical difference. This emphasizes the importance of contractility to provide the optimum afterload for the left ventricle already affected with low-preload due to MS. High EF and a good myocardial contractility has the potential to delay onset of symptoms. Finally, the high SPAP is the pathophysiological consequence of Group $2 \mathrm{PH}$ caused by high LA pressure and high PCWP in MS. It is not surprising that a smaller valve area initiates this pathological process sooner and higher pulmonary arterial pressures are recorded.

\section{LAA function}

LAA is the usual suspect of origin when a thromboembolic event occurs in the setting of mitral stenosis. Independent of mitral valve pathology, it has been reported that there is a negative correlation between LAA contraction velocity and mean pulmonary capillary wedge pressure ${ }^{25}$. Mitral stenosis is known to decrease LAA flow velocities by impairment of active and passive emptying functions of LAA both in sinus rhythm and in atrial fibrillation ${ }^{4,5}$. Although this functional detoriation is primarily due to hemodynamically elevated LA pressure, it is also possible that the chronic inflammatory progression of rheumatic disease directly affects LA and LAA ${ }^{5}$. An electron microscopy study showed atrial endocardial damage in mitral valve disease ${ }^{26}$. SEC and thrombus formation are some of the clinical consequences of functional impairment. LAA function was reported to recover after intervention or medical treatment. In patients with MS, following PMBV LAA functions improved and SEC or thrombus formation was less frequent in $L A A^{7,27}$. Additionally, with changes in loading conditions after heart failure treatment $L A A$ functions were reported to be improved ${ }^{28}$.

In various studies symptom duration ${ }^{29,30}, \mathrm{AF}^{30}, \mathrm{LA}_{\text {diameter }}{ }^{30}$, severity of $\mathrm{MS}^{31}$, age $\mathrm{e}^{29}$, dilatation of LAA with atrial body ${ }^{32}$ have been determined as predictors of SEC and thrombus in severe MS. It has been shown in previous studies that MR is protective for thrombus and SEC formation by decreasing stagnation in $\mathrm{LA}^{33}$ and that accompanied by severe MR decreases systemic embolism ${ }^{34}$. In our study, advanced age, larger LA diameter and smaller MVA were identified as predictors of thrombotic status in LAA in accordance with the literature. Severe MR patients were excluded however, patients with moderate MR did not show difference in LAA thrombus status. In a retrospective TEE study (1288 individual studies screened) exploring the relationship between SEC in the LA and CVA, SEC in SR was associated with high CVA prevalence independent of LA size and LAA function. One of the explanations for similar CVA rates in sinus rhythm and atrial fibrillation could be undetected paroxysmal atrial fibrillation as only ECG during TEE was reported. It is highly likely for patients with dilated left atrium to have paroxysmal AF episodes ${ }^{35}$.

Other predictors of the LAA thrombus can be listed as larger LA diameter, advanced age and smaller MVA in accordance with the literature ${ }^{28-30}$. Not only statistically but also the clinically significant variables were evaluated and it was shown that PFO as an independent predictor of LAA thrombotic status has an important contribution to the result. 


\section{Conclusion}

Combination of PFO with MS can be interpreted as a variation providing clinical benefit to the acquired valvular disease by alleviating the elevated LA pressure which is the corner stone of pathophysiology. Remaining asymptomatic for a longer periods and having less thrombosis constitute two separate entities of this benefit. We believe that, our study will also contribute to symptomatic benefit search with interventions targeted at LA pressure modulation.

\section{Limitations}

Retrospective data is the main restrictive feature of the study. Some parameters could not be reached or reanalyzed (left atrium volume index, right atrium volume index, right ventricule ejection fraction) from the TEE images recorded in the PACS system and the report records. Invasive MVA confirmation was not performed. Since the treatment options for MS are currently determined mainly with echocardiography, invasive hemodynamic pressure measurements were not available. SPAP was only evaluated by echocardiography, therfore it was not possible to distinguish between isolated postcapillary or combined pre and postcapillary $\mathrm{PH}$.

\section{Declarations}

\section{ACKNOWLEDGEMENTS}

We would like to extend our sincere thanks to Prof. Dr. Halil Ibrahim Tanboga. He provided us invaluable insight into statistical analyses and patience throughout the duration of this project. We'd also like to extend our gratitude to Dr. Suzan Hatipoglu Akpinar for constructive criticism and helpful contributions.

\section{References}

1.Hugenholtz PG,Ryan TJ,Stein SW,Abelmann WH. The spectrum of pure mitral stenosis. Hemodynamic studies in relation to clinical disability. Am J Cardiol 1962;10:773-84.

2.Mittnacht AJ,Fanshawe M,Konstadt S. Anesthetic considerations in the patient with valvular heart disease undergoing noncardiac surgery. Semin Cardiothorac Vasc Anesth 2008;12:33-59.

3.Lutembacher R. De la stenose mitrale avec communication interauriculaire. Arch Mal Coeur 1916; 9: 237-60.

4.Guler N,Demirbag R,Ozkara C, Beyhan Eryonucu, Ahmet Güneş, Mustafa Tuncer, et al. Clinical and echocardiographic predictors of left atrial appendage dysfunction in patients with rheumatic mitral stenosis in sinus rhythm. JASE 2004;17(8):819-23. 
5.Yoram Agmon, MD, Bijoy K. Khandheria, MD, Federico Gentile, MD, James B. Seward, MD. Echocardiographic Assessment of the Left Atrial Appendage. JACC 1999;34:1867-77.

6.Z Gölbaşi,D Ciçek, A Canbay,O Uçar, H Bayol,S Aydogdu. Left Atrial Appendage Function in Patients With Mitral Stenosis in Sinus Rhythm. Eur J Echocardiogr. 2002;3(1):39-43.

7.Lin JM, Hsu KL, Hwang JJ, Li YH, Tseng YZ. Effects of percutaneous mitral valvuloplasty on left atrial appendage flow velocity. Am J Cardiol 1995;76:609-11.

8. Hagen PT, Scholz DG, Edwards WD. Incidence and size of patent foramen ovale during the first 10 decades of life: an autopsy study of 965 normal hearts. Mayo Clin Proc 1984;59:17.

9.Chau-Chung Wu,Wen-Jone Chen,Ming-Fong Chen,Chiau-Suong Liau, Shu-Hsun Chu, Yuan-Teh Lee. Leftto-right shunt through patent foramen ovale in adult patients with left-sided cardiac lesions:A transesophageal echocardiographic study. Am Heart J 1993;125:1369.

10.Kaye D, Shah SJ, Borlaug BA, Finn Gustafsson, Jan Komtebedde, Spencer Kubo, et al. Effects of an interatrial shunt on rest and exercise hemodynamics:results of a computer simulation in heart failure. $J$ Card Fail. 2014;20:212-221.

11.The Helsinki Declaration of the World Medical Association(WMA).Ethical Principles of Medical Research Involving Human Subjects. Pol Merkur Lekarski 2014;36(215):298-301.

12. Helmut Baumgartner, Judy Hung, Javier Bermejo, John B. Chambers, Arturo Evangelista, Brian P. Griffin, et al. Echocardiographic assessment of valve stenosis: EAE/ASE recommendations for clinical practice. Eur J Echocardiogr 2009;10(1):125.

13.Lancellotti P, Tribouilloy C, Hagendorff A, Bogdan A Popescu, Thor Edvardsen, Luc A Pierard, et al. Recommendations for the echocardiographic assessment of native valvular regurgitation: an executive summary from the European Association of Cardiovascular Imaging. Eur. Heart J.: Cardiovasc. Imaging 2013;14:611-644.

14.Silvestry FE, Cohen MS, Armsby LB, Nitin J Burkule, Craig E Fleishman, Ziyad M Hijazi et al. Guidelines for the echocardiographic assessment of atrial septal defect and patent foramen ovale. JASE 2015;28:910-58.

15.Kerut EK, Norfleet WT, Plotnick GD, Giles TD. Patent foramen ovale:A review of associated conditions and the impact of physiological size. JACC 2001;38:613.

16.Kurzyna M, Dabrowski M, Bielecki D, Anna Fijalkowska, Piotr Pruszczyk, Grzegorz Opolski et al. Atrial septostomy in treatment of end-stage right heart failure in patients with pulmonary hypertension. Chest 2007;131:977-983. 
17.Lehner A, Schulze-Neick I, Fischer M, Silvia Fernandez-Rodriguez, Sarah Ulrich, Nikolaus A. Haas, et al. The Creation of an Interatrial Right-To-Left Shunt in Patients with Severe, Irreversible Pulmonary Hypertension: Rationale, Devices, Outcomes. Curr Cardiol Rep. 2019;21(5):31.

18.Steinbrunn W, Cohn KE, Selzer A. Atrial septal defect associated with mitral stenosis. The Lutembacher syndrome revisited. Am J Med 1970;48:295-302.

19.Crawford MH. latrogenic Lutembacher's syndrome revisited. Circulation 1990;81:1422-1424.

20.Cequier A, Bonan R, Serra A, Dyrda I, Crepeau J, Dethy M, Waters D. Left-to-right atrial shunting after percutaneous mitral valvuloplasty: Incidence and long-term hemodynamic follow-up. Circulation 1990;81:1190-1197.

21.Reddy YNV, Olson TP, Obokata M, Melenovsky V, Borlaug BA. Hemodynamic correlates and diagnostic role of cardiopulmonary exercise testing in heart failure with preserved ejection fraction. JACC HF 2018;6:665-75.

22.Dorfs S, Zeh W, Hochholzer W, Nikolaus Jander, Rolf-Peter Kienzle, Burkert Pieske et al. Pulmonary capillary wedge pressure during exercise and longterm mortality in patients with suspected heart failure with preserved ejection fraction. EHJ 2014;35:3103-12.

23.Hasenfuß G, Hayward C, Burkhoff D, Frank E Silvestry, Scott McKenzie, Finn Gustafsson,

et al. A transcatheter intracardiac shunt device for heart failure with preserved ejection fraction (REDUCE LAP-HF): a multicentre, open-label, single-arm, phase 1 trial. Lancet 2016;387:1298-304.

24.Obokata M, Reddy YNV, Shah SJ, David M Kaye, Finn Gustafsson, Gerd Hasenfuß, et al. Effects of interatrial shunt on pulmonary vascular function in heart failure with preserved ejection fraction. JACC 2019;74(21):2539-2550.

25.Tabata T, Oki T, Fukuda N,Arata Luchi, Kazuyo Manabe, Yoshimi Kageji et al. Influence of left atrial pressure on left atrial appendage flow velocity patterns in patients in sinus rhythm. JASE 1996;9:857-64.

26.Goldsmith I, Kumar P, Carter P, Andrew D. Blann, Ramesh L. Patel, Gregory Y.H. Lip. Atrial endocardial changes in mitral valve disease: a scanning electron microscopy study. Am. Heart J. 2000;140 (5):777784.

27.Porte JM, Cormier B, lung B, E Dadez, C Starkman, O Nallet, et al. Early assessment by transesophageal echocardiography of left atrial appendage function after percutaneous mitral commissurotomy. Am J Cardiol 1996;77:72- 6.

28.Ito T, Suwa M, Kobashi A, Yagi H, Hirota Y, Kawamura K. Influence of altered loading conditions on left atrial appendage function in vivo. Am J Cardiol 1998;81,1056-1059. 
29.Goswami KC, Narang R, Bahl V K, Talwar KK, Manchanda SC. Comparative evaluation of transthoracic and transesophageal echocardiography in the detection of left atrial clot before percutaneous transvenous mitral commissurotomy: do all patients need transesophageal examination? Int J Cardiol 1997;62:237-49.

30.Goswami KC, Yadav R, Rao MB, Bahl VK, Talwar KK, Manchanda SC. Clinical and echocardiographic predictors of left atrial clot and spontaneous echo contrast in patients with severe rheumatic mitral stenosis: a prospective study in 200 patients by transesophageal echocardiography. Int $\mathrm{J}$ Cardiol 2000;73:273-9.

31.Kronzon I, Tunick PA, Glassman E, Slater J, Schwinger M, Freedberg RS. Transesophageal echocardiography to detect atrial the presence of left atrial spontaneous echo contrast clot in candidates for percutaneous transseptal mitral balloon valvuloplasty. JACC 1990;16:1320-2.

32.Fatkin D, Kelly RP, Feneley MP. Relations between left atrial appendage blood flow velocity, spontaneous echocardiographic contrast and thromboembolic risk in vivo. JACC 23(4):961-969.

33.Ozkan M, Kaymaz C, Kirma C, A Civelek, A R Cenal, C Yakut, et al. Predictors of left atrial thrombus and spontaneous echo contrast in rheumatic valve disease before and after mitral valve replacement. Am J Cardiol 1998;82:1066-1070.

34.Kaymaz C,Ozdemir N,Erentug V,Sismanoglu M,Yakut C,Ozkan M. Location, Size, and Morphologic Characteristics of Left Atrial Thrombi as Assessed by Transesophageal Echocardiography in Relation to Systemic Embolism in Patients With Rheumatic Mitral Valve Disease. Am J Cardiol 2003;15;91(6):765-9.

35-Saihari Sadanandan, Mark V. Sherrid. Clinical and Echocardiographic Characteristics of Left Atrial Spontaneous Echo Contrast in Sinus Rhythm. JACC 2000;35:1932- 8

\section{Tables}

Table 1: Demographic characteristics of patients included in the study 


\begin{tabular}{|l|l|}
\hline Age (years) & $48 \pm 12$ \\
\hline Gender(male), n (\%) & $75(23,4)$ \\
\hline Hypertension, n (\%) & $76(23,8)$ \\
\hline Diabetes Mellitus, n (\%) & $28(8,8)$ \\
\hline Patent foramen ovale, n (\%) & $34(10,6)$ \\
\hline Sinus rythym on ECG, n (\%) & $190(59,4)$ \\
\hline MVA(PHT),cm ${ }^{2}$ & $1,15 \pm 0,19$ \\
\hline MVA(PLN),cm ${ }^{2}$ & $1,15 \pm 0,19$ \\
\hline MVA 3-MPR,cm ${ }^{2}$ & $1,09 \pm 0,23$ \\
\hline Maximum gradient of MV,mmHg & $21,(\mathrm{IQR}: 26-17)$ \\
\hline Mean gradient of MV,mmHg & $13,(\mathrm{IQR}: 16-9)$ \\
\hline Wilkins Score & $8,(\mathrm{IQR}: 9-7)$. \\
\hline SPAP,mmHg & $45,(\mathrm{IQR}: 55-35)$ \\
\hline LA diameter,mm & $45,(\mathrm{IQR}: 50-42)$ \\
\hline LVEF (\%) & $60,(\mathrm{IQR}: 65-60)$ \\
\hline Mild MR ,n (\%) & $139(43,4)$ \\
\hline Mild to moderate MR,n (\%) & $98(30,6)$ \\
\hline Mild TR,n (\%) & $124(38,8)$ \\
\hline Moderate TR,n (\%) & $147(45,9)$ \\
\hline Severe TR,n (\%) & $27(8,4)$ \\
\hline Mild AR,n (\%) & $88(27,5)$ \\
\hline Mild to moderate AR,n $\%)$ & $103(32,2)$ \\
\hline ASA use,n (\%) & $81(25,3)$ \\
\hline Warfarin use,n (\%) & $88(27,5)$ \\
\hline
\end{tabular}

MVA:mitral valve area, MV:mitral valve, MR:mitral regurgitation, TR:tricuspid regurgitation, AR:aortic regurgitation, SPAP:sistolic pulmonary artery pressure, LA:left atrium, LVEF:left ventricule ejection fraction, ECG:electrocardiography, ASA:acetylsalicylic acid, 3DMPR: three dimensional multiplanar reconstruction, PHT:pressure half time, PLN:planimetric, IQR:interquartile range

\section{Table 2:}

Multivariable ordinary least square model for the predictors of mitral valve area 


\begin{tabular}{|l|l|l|l|}
\hline & Regression coefficient & CI 95(\%) & P value \\
\hline SPAP (from 35 to $55 \mathrm{mmhg}$ ) & -0.050 & $-0.105,-0.0046$ & 0.006 \\
\hline LVEF (from 60 to $65 \%)$ & -0.045 & $-0.092,-0.0003$ & 0.0015 \\
\hline PFO (yes) & -0.119 & $-0.189,-0.0502$ & 0.0008 \\
\hline Age (from 39.7 to 56 years) & 0.011 & $-0.050,0.073$ & 0.693 \\
\hline LA diameter (from 42 to 49 mm) & -0.018 & $-0.080,0.044$ & 0.858 \\
\hline Gender (male) & -0.011 & $-0.069,0.045$ & 0.684 \\
\hline $\begin{array}{l}\text { MR grade } \\
\text { No } \\
\text { Mild } \\
\text { Mild to moderate }\end{array}$ & -0.016 & $-0.073,0.040$ & \\
& $\begin{array}{l}\text { Ref. } \\
\text { Ref. }\end{array}$ & 0.120 \\
& 0.042 & $-0.009,0.094$ & \\
& & & \\
\hline
\end{tabular}

PFO:patent foramen ovale, MR:mitral regurgitation, SPAP:sistolic pulmonary artery pressure, LA:left atrium, LVEF:left ventricular ejection fraction

Table 3: Multivariable proportional odds logistic regression model for the predictors of LAA thrombotic status

\begin{tabular}{|c|c|c|}
\hline & Odds ratio and $95^{\circ}$ & $\mathrm{I} P$ value \\
\hline Age (from 39.8 to 56 years) & $2.36(1.51-3.71)$ & 0.001 \\
\hline MVA (from 1.0 to $1.3 \mathrm{~cm}^{2}$ ) & $0.56(0.37-0.86)$ & 0.018 \\
\hline LA Diameter (from 42 to $50 \mathrm{~mm}$ ) & $1.99(1.37-2.88)$ & 0.001 \\
\hline LVEF (from 60 to 65 ) & $0.94(0.54-1.64)$ & 0.325 \\
\hline \begin{tabular}{|} 
Baseline antithrombotic drug \\
No \\
Antiplatelet \\
Anticoagulant
\end{tabular} & $\begin{array}{l}0.86(0.46-1.62) \\
0.76(0.40-1.43) \\
\text { Ref. }\end{array}$ & 0.696 \\
\hline Gender (male) & $0.73(0.39-1.35)$ & 0.317 \\
\hline \begin{tabular}{|l} 
MR grade \\
No \\
Mild \\
Mild to moderate
\end{tabular} & $\begin{array}{l}0.89(0.46-1.72) \\
\operatorname{Ref} \\
0.708(0.37-1.30)\end{array}$ & 0.536 \\
\hline PFO (yes) & $0.38(0.15-0.94)$ & 0.036 \\
\hline
\end{tabular}

PFO: patent foramen ovale, MR: mitral regurgitation, MVA: mitral valve area, LA: left atrium, LVEF: left ventricular ejection fraction 


\section{Figures}
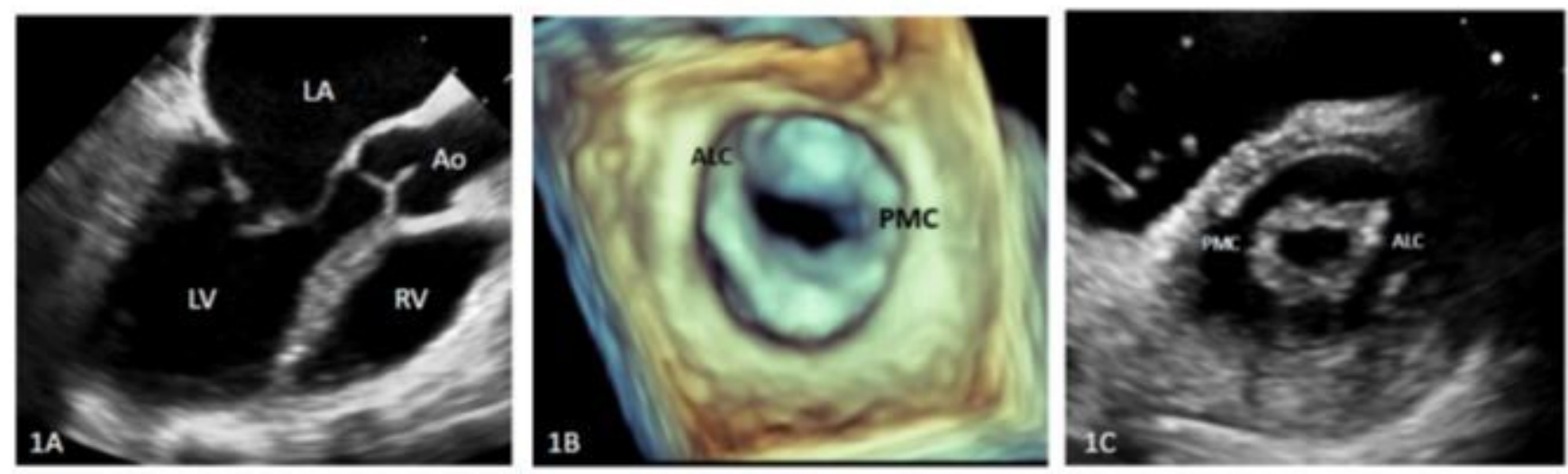

\section{Figure 1}

1A:Left ventricle outflow view of transesophageal echocardiography shows diastolic restriction of thickened mitral leaflets, 1B: 3D zoom image of rheumatic mitral valve from left atrial aspect, 1C: Parasternal short axis view of mitral valve, LA: left atrium, LV: left ventricle, Ao: aorta, RV: right ventricle, ALC: anterolateral commissure, PMC: posteromedial commissure
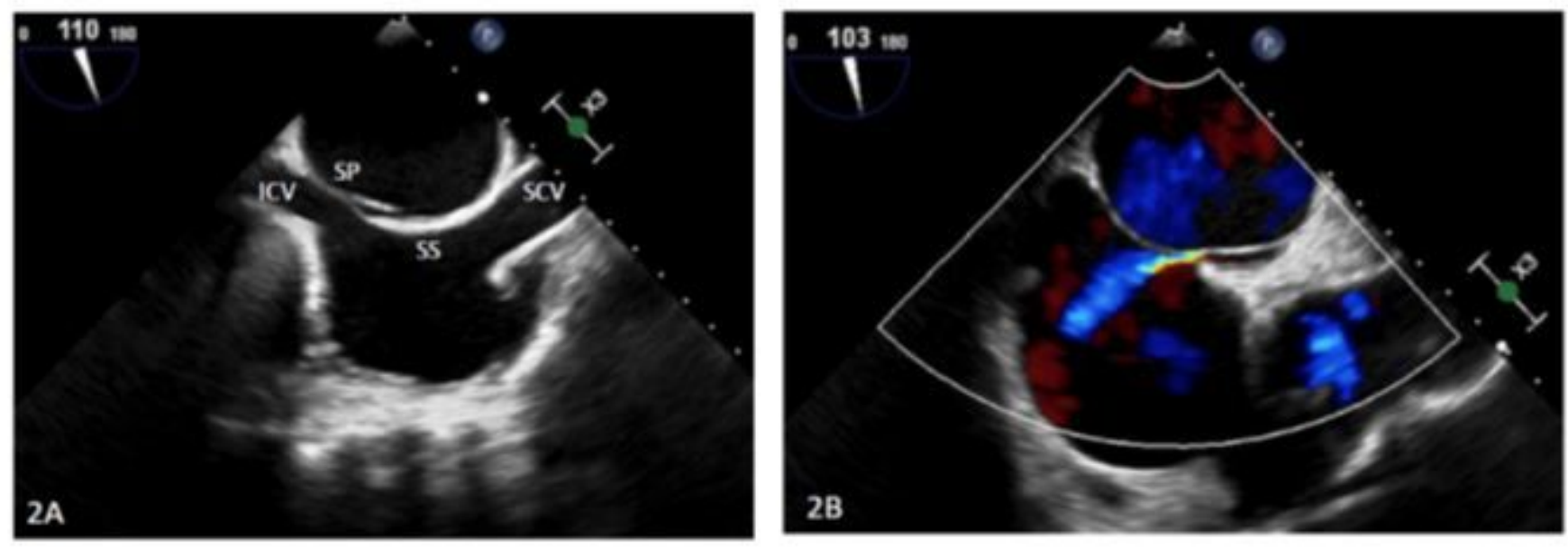

\section{Figure 2}

2A:Patent foramen ovale tunnel at bicaval view, 2B: Left-to-right shunt through patent foramen ovale, IVC: inferior vena cave, SVC: superior vena cava, SP: septum primum, SS: septum secundum 